\title{
Study on Compression of ICF Fuel in Rocket Model
}

\author{
Vijay Kumar Jha ${ }^{1,2}$ \\ ${ }^{1}$ Central Department of Physics, Tribhuvan University, Kathmandu, Nepal \\ ${ }^{2}$ Amrit Campus, Tribhuvan University, Thamel, Kathmandu, Nepal
}

Email address:

jhav7050@gmail.com

To cite this article:

Vijay Kumar Jha. Study on Compression of ICF Fuel in Rocket Model. American Journal of Physics and Applications. Vol. 5, No. 6, 2017, pp. 95-98. doi: 10.11648/j.ajpa.20170506.14

Received: August 21, 2017; Accepted: September 11, 2017; Published: October 20, 2017

\begin{abstract}
Compression of Inertial Confinement Fusion (ICF) fuel as required by Lawson Criterion has been of immense value in ICF studies. In this work, the order of compression has been studied on Rocket Model because a high-order reaction force responsible for compression may be seen to act as a rocket motion. It has been seen that the order of compression of lighter fuel such as D-T may be more effective if irradiated by high power Nd laser. The shocks produced as the reaction (Rocket effect) to the surface ablation generated by pulsed laser beams, compress the fuel which is estimated to be effective when the ratio of initial mass to the accelerated one is of the order of 5 . The maximum achievable compression by a single strong shock is not more than 4 for a monatomic gas. For weak coalescing shocks to achieve adiabatic compression, the ablation efficiency is found to be maximum when target velocity equals nearly twice the ablation velocity. In such a case, the implosion efficiency of Rocket Model is found to be about 67 percent; neglecting heat loss.
\end{abstract}

Keywords: Inertial Confinement Fusion (ICF), Lawson Criterian, Compression, Ablation, Implosion Efficiency, Rocket Model, Shock Wave, Mach Number, Fermi Degenerate Adiabat

\section{Introduction}

Controlled nuclear fusion research has the potential to meet global energy requirement of the future. Though difficult technologically, there are two schemes for fusion research. Large resources in the form of seawater with negligible radiation hazard associated with these schemes make thermonuclear fusion research an important one for industrial development of the world. The difficulties involved in igniting and controlling the fusion are formidable because scattering of positively charged nuclei is several times more probable than fusion event. Inertial confinement fusion (ICF) scheme is one of the processes in which high power lasers not only produce plasma but also create suitable situation for confinement since the mass of the ions in the core becomes too high with respect to the electrons in the corona. Lawson criterion has been the first and foremost basis of ICF scheme according to which the fuel requires high compression of the order $10^{4}$.

As required by the criterion the compression plays a key role in increasing the density of fuel to an optimum range. If the fuel pellet is compressed to sufficient densities, it will burn so rapidly that appreciable fusion energy will be released before it can blow apart. The present study is aimed at 'compression' of ICF fuel the situation of which may be studied like the motion of a rocket [1, 2, 3]. High power lasers create such situation in ICF scheme.

\section{Method (Theory)}

Deuterium-Tritium fuel placed in an infinitely thin spherical shell if irradiated by a high power Nd laser from all possible sides the surface of the pellet heats, ionizes and ablates off to surround the pellet in a cloud or 'corona' of lowdensity plasma. As the ablation of the surface continues shock fronts as reaction are formed that converge inward pushing cold D-T fuel ahead of it to higher and higher densities along the "Fermi degenerate adiabat". These inward pushing shock waves compress the fuel to high order and may be considered as in good analogy with the motion of a rocket $[2,3]$ since ablation $[4,5,6,7]$ and compression act in opposite direction like rocket motion. 
Assume that a high aspect ratio shell can be adequately represented by an infinitely thin mass shell of mass $M_{s}$ and radius $R(t)$. We suppose that the ablation material density $\rho_{a}$ and ablation velocity $\mathrm{v}_{a}$ (relative to the moving shell surface) are constant. Also, the shell is assumed to contain an adiabatic fuel $\left(P_{F} V^{\gamma}=\right.$ constant $)$ that eventually compress to a pressure sufficient to reverse the inward motion of the imploding shell. Then, two forces drive the implosion of the shell:

1) due to reactive force of the ablating material (the "rocket effect"), and

2) due to the ablation pressure caused by the deposition and transport of driver energy as heat into the ablation surface

Thus, using Newton's law, the equation of motion for this model can be written as

$$
\frac{d}{d t}\left(M_{S} \dot{R}\right)=F_{\text {rocket }}+F_{P_{a}}=\left(\dot{R}+\mathrm{v}_{a}\right) \dot{M}_{s}+4 \pi R^{2}\left(P_{F}-P_{a}\right)
$$

The shell losses mass because of ablation at a rate

$$
\dot{M}_{s}=-4 \pi R^{2} \rho_{a} \mathrm{v}_{a}
$$

where

$$
\dot{R}=\frac{d R}{d t} \text { and } \dot{M}_{s}=\frac{d M_{s}}{d t}
$$

If we introduce the characteristic implosion time [2] to analyze this model as

$$
\tau=\left(\frac{M_{0}}{4 \pi R_{0} \rho_{a}}\right)^{\frac{1}{2}} \frac{1}{c_{a}}
$$

We can write equation (1) in dimensionless form as

$$
\eta \frac{d^{2} y}{d t^{2}}=-\left(M^{2}+1-\beta y^{-3 \gamma}\right) y^{2}
$$

where

$$
\eta(t)=\frac{M_{S}(t)}{M_{0}}=1-\int_{0}^{t} M \alpha y^{2} d t^{\prime} ; y(t)=\frac{R(t)}{R_{0}} ; \quad t^{\prime}=\frac{t}{\tau} ;
$$
initial shell mass $\left(M_{0}\right)=4 \pi R_{0}{ }^{2} \delta \rho_{\text {shell }}=4 \pi \mathrm{R}_{0}{ }^{2} \delta \rho_{\text {shell }}$;

$\operatorname{Mach}$ number $(M)=\frac{\mathrm{v}_{a}}{C_{a}} ; c_{a}=\sqrt{\frac{P_{a}}{\rho_{a}}} ;=\frac{P_{0}}{P_{a}} ; \alpha=\left(\frac{R_{0}}{\delta} \frac{\rho_{a}}{\rho_{\text {solid }}}\right)^{\frac{1}{2}}$ and $\delta=\Delta R$.

Equation (2) has been numerically integrated for initial conditions: $\eta(0)=1, y(0)=1, y(0)^{*}=0$ for various choices of parameters $M, \alpha$ and $\beta$. Before examining these solutions, it is useful to compute the various energies associated with this model. If we multiply by $\dot{R}$ and integrate, we find [2]

$$
E_{K E}^{\text {shell }}=\frac{3}{2} E_{0}\left(\frac{d y}{d t^{\prime}}\right)^{2} \eta=\frac{1}{2} M_{S} R^{2}=\text { Shell kinetic energy }
$$

where $E_{0}=\frac{4 \pi R_{0}{ }^{3} P_{a}}{3}$ is the energy needed to fill the original shell volume at the ablation pressure. Similarly, we can solve [2] for

$$
\begin{aligned}
& E_{K E}^{\text {ablation }}=\frac{1}{2} \int_{0}^{t}\left[\dot{M}_{s}\left(\dot{R}+\mathrm{v}_{a}\right)^{2}\right] d t \\
& =\frac{3}{2} E_{0} \int_{0}^{t} \dot{\eta}\left(\dot{y}+\frac{M_{s}}{\alpha}\right) d t^{\prime} \\
& \text { = blow off kinetic energy } \\
& E_{m e c h}=-\int_{0}^{t} 4 \pi R^{2} P_{a} \dot{R} d t=-3 E_{0} \int_{0}^{t} y^{2} \dot{y} d t^{\prime} \\
& \text { = mechanical energy due to ablation presssure } \\
& E_{\text {exh }}=\frac{1}{2} \int_{0}^{t} \dot{M}_{s} \mathrm{v}_{a}^{2} d t=\frac{3}{2} E_{0} \frac{M^{2}}{\alpha^{2}} \int_{0}^{t} \dot{\eta} d t^{\prime} \\
& =\text { Rocket exhaust energy }
\end{aligned}
$$

The energy balance can be noticed as

$$
\begin{aligned}
\text { input energy } & \rightarrow E_{K . E .}^{\text {shell }}+E_{K . E .}^{\text {ablation }}=E_{\text {mech }}+E_{\text {exh }} \\
& \rightarrow \text { compression }
\end{aligned}
$$

Detailed examination of this model reveals that the transfer of energy from the mechanical input (ablation pressure) to the shell kinetic energy is quite efficient.

The expression for hydrodynamic or ablation efficiency $\eta_{h}$ and implosion efficiency $\eta_{\text {implosion }}$ are obtained as

$$
\eta_{h}=\left(\frac{v}{v_{a}}\right)^{2}\left[\exp \left(\frac{V}{v_{a}}\right)-1\right]^{-1}
$$

and

$$
\eta_{\text {implosion }}=\frac{x \ln ^{2} x}{(1-x)}
$$

where $x \equiv \frac{M_{s}}{M_{0}}$

Here $M_{s}, M_{0}, V, \mathrm{v}_{a}$ are respectively rocket mass(fuel mass), initial mass, rocket speed(implosion speed), exhaust speed(ablation speed).

The efficiency with which the fuel can be accelerated to the required implosion velocity is called the implosion efficiency. It can be estimated by using a simple analogy, which models the imploding fuel as a rocket. The Proposed ICF capsules are imploded by ablation of an outer shell [Figure 1]. 


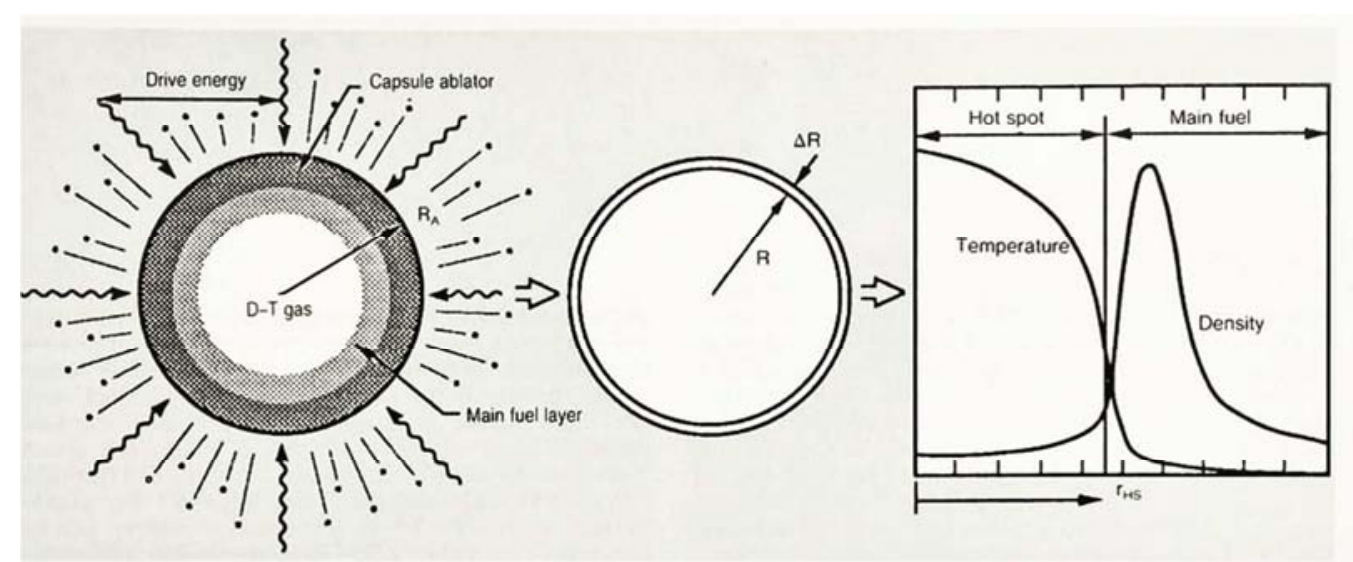

Figure 1. Proposed ICF Capsules [3] are imploded by ablation of an outer shell. ignition occurs in a central hot spot, and fusion burn propagates out through the main mass of D-T Fuel.

\section{Results and Discussion}

In the present theoretical study of compression, it is found that the maximum laser energy transfer in coronal region occurs when the ratio of initial mass of the pellet to the ablated mass is of the order of 5 if one expects better compression. The order of compression as a function of Mach number is plotted in Figure 2, for a monatomic gas. It is clear from the figure that the maximum achievable compression by a single strong shock $[8,9,10]$ cannot be more than 4 which supports the use of a series of weak shocks instead of using a single strong shock to optimize compression.

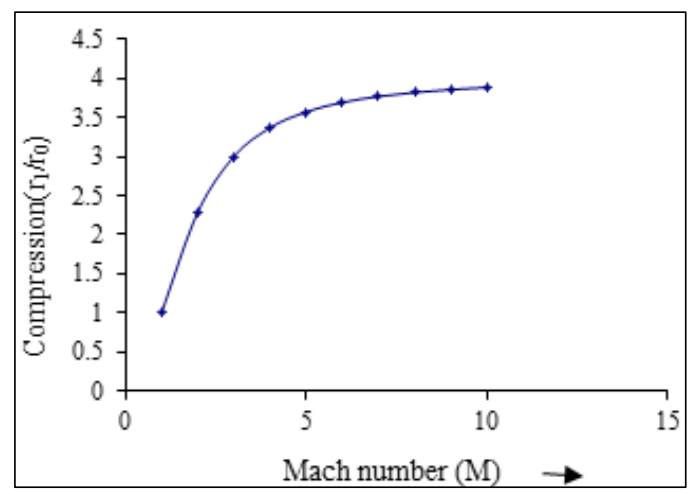

Figure 2. Variation of Compression with Mach No.

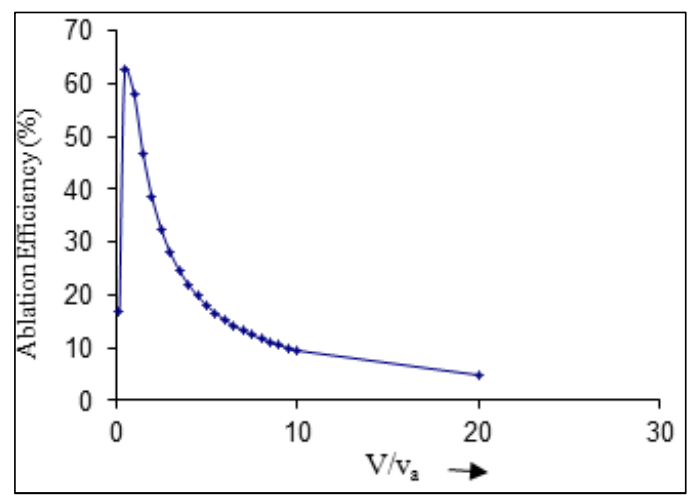

Figure 3. Ablation Efficiency as a Function of Velocity Ratio.

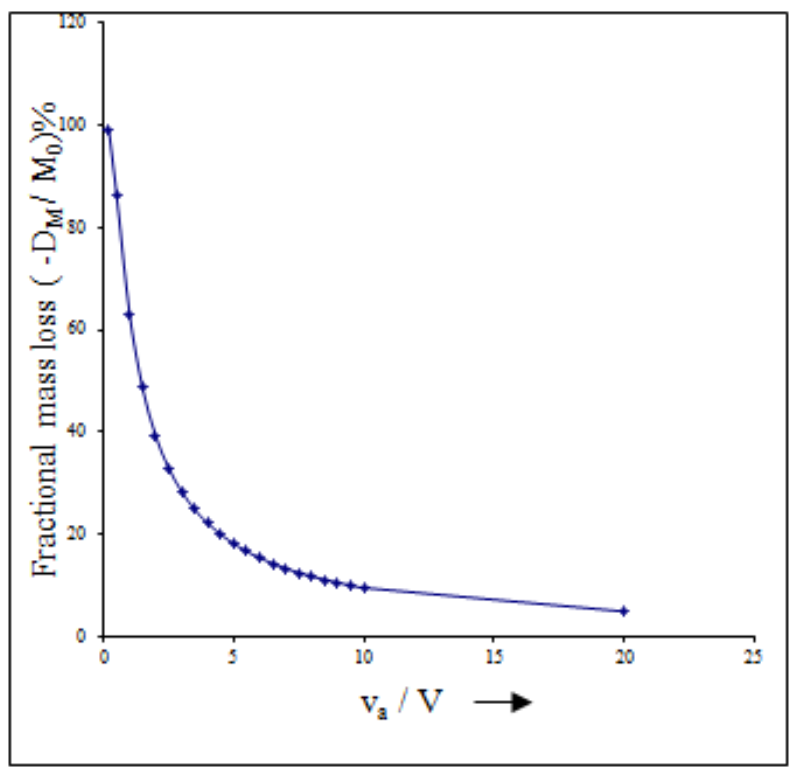

Figure 4. Variation in Frazctional Mass Loss as a Function of Velocity Ratio.

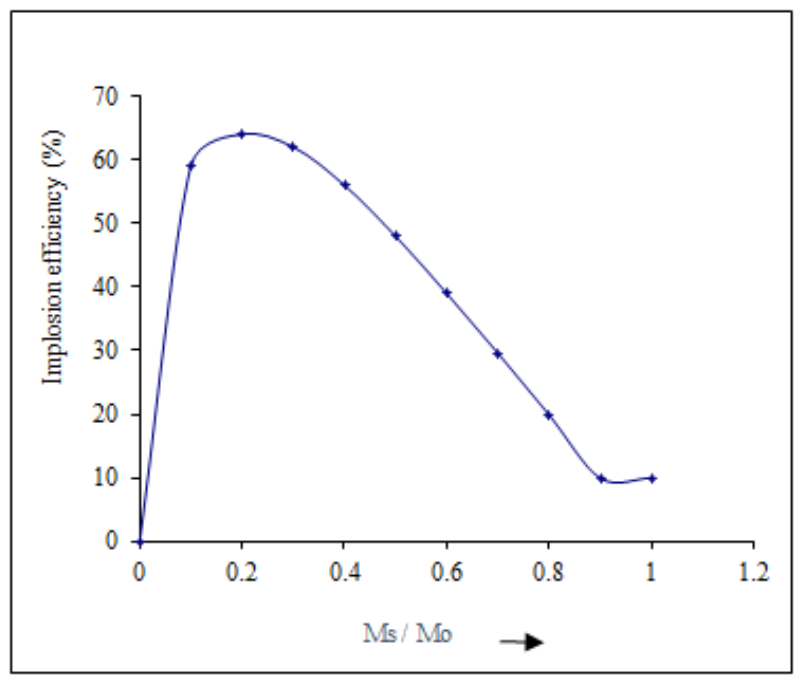

Figure 5. Variation in Implosion Efficiency of Rocket Model with $M_{s} / M_{o}$

The advantages of compression are partially compensated 
by energy losses associated with escaping ablated material. These losses are related to implosion and blow off velocities by rocket equation. The ablation efficiency $\eta_{h}$, approximately given by the ratio of kinetic energy of accelerated target to the absorbed laser energy, is plotted against velocity ratio (ablation velocity/target velocity) in Figure 3, in accordance with equation (3). The velocity ratio is again plotted against fractional mass loss in Figure 4. From these figures it is clear that, for smaller fractional mass loss

$$
\eta_{h} \approx V / \mathrm{v}_{a} \approx \Delta m / M_{0}
$$

and that ablation efficiency is maximum when $\mathrm{v}_{a}=\frac{V}{2}$.

The implosion efficiency, i.e. the efficiency with which the fuel can be accelerated to the required implosion velocity (rocket velocity) can be estimated by plotting mass ratio in accordance with equation (4). The implosion efficiency $\eta_{\text {implosion }}$ is also plotted against the mass ratio in Figure 5 on the basis of equation (4). It is seen that $V / \mathrm{v}_{a}$ decreases exponentially with increase in $M_{s} / M_{0}$. It is also observed that for good efficiency, $M_{s} / M_{0} \approx 0.2$ for which we need $\mathrm{V} \sim 2 \mathrm{v}_{a}$. But using an earlier approximation of $V \sim 3 \times 10^{7} \mathrm{~cm} / \mathrm{s}$, we find that the ablation velocity should be $\mathrm{v}_{a} \sim 1.5 \times 10^{7} \mathrm{~cm} / \mathrm{s}$. This implosion efficiency indicates that only about $10 \%$ of the driver energy can ultimately end up in the fuel. One milligram of mass travelling at a velocity of $3 \times 10^{7} \mathrm{~cm} / \mathrm{s}$ has a kinetic energy of $45 \mathrm{~kJ}$. Therefore without accounting for any other loss mechanisms such as the acceleration of non-fuel material, we require $450 \mathrm{~kJ}$ of driver input energy. Of course this is not strictly correct because not all of the fuel mass need be accelerated to $3 \times 10^{7} \mathrm{~cm} / \mathrm{s}$ to achieve final shock velocities of $3 \times 10^{7} \mathrm{~cm} / \mathrm{s}$. Thus a proper shock sequencing is required to achieve ignition.

\section{Conclusions}

A simple rocket model thus indicates that it is the ablation pressure that influences the fuel compression most strongly and the energy delivered to the shell from the mechanical ablation pressure is the most efficient energy transfer mechanism. The ablation efficiency reaches a maximum value when target velocity equals nearly twice the ablation velocity and in this situation, implosion efficiency of rocket model tends to be about $67 \%$ if heat loss is not taken into account.

\section{References}

[1] J. Nuckolls, L. Wood, A. Thiessen and G Zimmerman, Nature 239, 139 (1972).

[2] J. J. Duderstadt and G. A. Mosses, ICF, John Wiley and sons, (1982).

[3] J. D. Lindl, R. L. McCrory and E. Michael Campbell, Physics Today, American Institute of Physics, 32-35 (September 1992).

[4] R. Betti and O. A. Hurricane, Nature Physics 12, 435-448 (2016).

[5] E. L. Vold, R. M. Rauenzahn, C. H. Aldrich, K. Molvig, A. N. Simakov, and B. M. Haines less, Physics of Plasmas 24, 042702 (2017).

[6] R. S. Craxton, K. S. Anderson, T. R. Boehly, V. N. Goncharov, D. R. Harding, Physics of Plasmas 22, 110501 (2015).

[7] S. M. Alastair, P. Shon, Kevin L. B. Kevin, M. C. Peter, F. Jonathan, R. D. Thomas, J. W. Kuang-Jen, L. K. Margaret, E. S. Michael, F. Mike, N. Abbas, A. H. Omar, Journal of Physics: Conference Series, 717, 012038 (2016).

[8] B. H. Ripin, R. Decoste, S. P. Obenschein, S. E. Bonder, E. A. McLean, F. C. Young, R. R. Whitlock, C. M. Armstrong, J. Grun, J. A. Stamper, S. H. Gold D. J. Nagel, R. H. Lehmberg and J. M. McMahon, Physics Fluids, 23, 1012 (1980).

[9] D. B. Schaeffer, W. Fox, D. Haberberger, G. Fiksel, A. Bhattacharjee, D. H. Barnak, S. X. Hu, and K. Germaschewski Phys. Rev. Lett. 119, 2-14 (July 2017).

[10] R. P. Drake, P. A. Keiter, C. C. Kuranz, G. Malamud, M. Manuel, C. A. Di Stefano, E. J. Gamboa, C. M. Krauland, M. J. MacDonald, W. C. Wan, R. P. Young, D. S. Montgomery, C. Stoeckl and D. H. Froula, Journal of Physics: Conference Series, 688 (1) (2016). 